EPJ Web of Conferences 106, 01008 (2016)

DOI: $10.1051 /$ epjconf/201610601008

(C) Owned by the authors, published by EDP Sciences, 2016

\title{
Fast Neutron Transport in the Biological Shielding Model and Other Regions of the VVER-1000 Mock-Up on the LR-0 Research Reactor
}

\author{
Michal Košt'ál ${ }^{1}$, Ján Milčák ${ }^{1}$, František Cvachovec ${ }^{2}$, Bohumil Jánský ${ }^{1}$, Vojtěch Rypar ${ }^{1}$, \\ Vlastimil Juř́iček ${ }^{1}$, Evžen Novák ${ }^{1}$, Alexander Egorov ${ }^{3}$, and Sergey Zaritskiy ${ }^{3}$ \\ ${ }^{1}$ Research Center Řež 1td., 25068 Husinec-Řež 130, Czech Republic \\ ${ }^{2}$ University of Defence, Kounicova 65, 66210 Brno, Czech Republic \\ ${ }^{3}$ NRC Kurchatov Institute, 1 Kurchatov Sq., Moscow 123182, Russia
}

\begin{abstract}
A set of benchmark experiments was carried out in the full scale VVER1000 mock-up on the reactor LR-0 in order to validate neutron transport calculation methodologies and to perform the optimization of the shape and locations of neutron flux operation monitors channels inside the shielding of the new VVER-1000 type reactors. Compared with previous experiments on the VVER-1000 mock-up on the reactor LR-0, the fast neutron spectra were measured in the extended neutron energy interval $(0.1-10 \mathrm{MeV})$ and new calculations were carried out with the MCNPX code using various nuclear data libraries (ENDF/B VII.0, JEFF 3.1, JENDL 3.3, JENDL 4, ROSFOND 2009, and CENDL 3.1). Measurements and calculations were carried out at different points in the mock-up. The calculation and experimental data are compared.
\end{abstract}

\section{Introduction}

The investigations on the shielding models were developed on the LR-0 research reactor for benchmarking the calculations of the neutron and gamma distributions in a VVER-1000 concrete shielding. Such calculations are carried out particularly for the optimization of the shape and locations of neutron flux operation monitors channels inside the shielding of the new VVER type reactors.

In order to perform the mentioned optimization and validate corresponding calculation methodologies a set of benchmark experiments was carried out in the full scale VVER-1000 mockup on the reactor LR-0. The results of the experimental and calculation analysis of the fast, epithermal and thermal neutrons and gammas distributions in the shielding models were presented in [1]. After this work the fast neutron spectra measurements were repeated in the extended neutron energy interval at different points of the mock-up including the shielding model and new calculations were performed with the MCNPX code using various nuclear data libraries (ENDF/B VII.0, JEFF 3.1, JENDL 3.3, JENDL 4, ROSFOND 2009, and CENDL 3.1), which were processed using NJOY code.

This is an Open Access article distributed under the terms of the Creative Commons Attribution License 2.0, which permits unrestricted use, distribution, and reproduction in any medium, provided the original work is properly cited. 


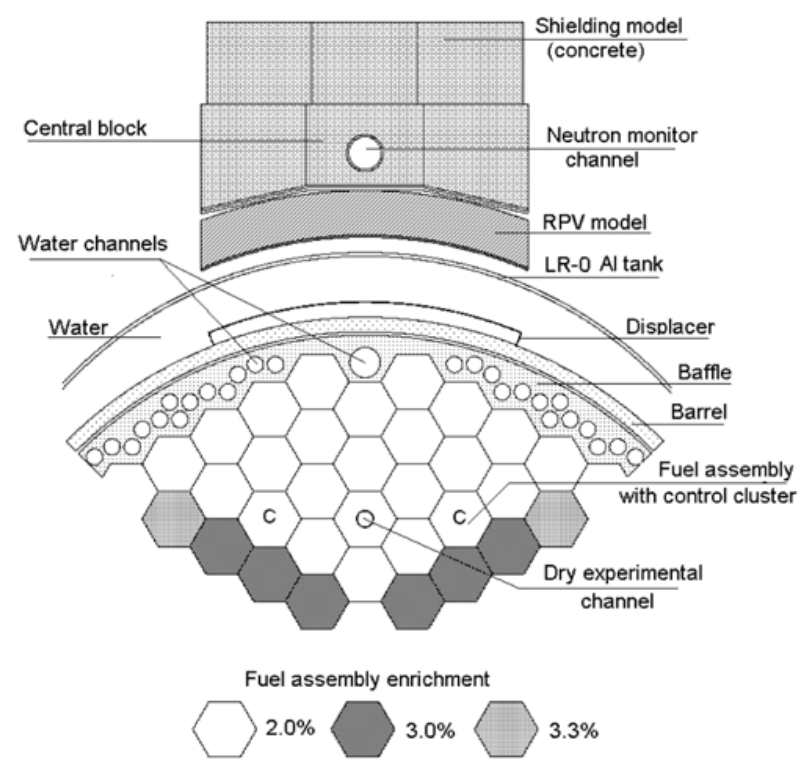

Figure 1. The layout of the VVER-1000 mock-up on the reactor LR-0 (top view).

The main task of this report is to present measured fast neutron spectra in the neutron energy interval 0.1-10 MeV and to compare these measurements with calculations using MCNPX and different data libraries.

\section{Mock-up Arrangement}

The experiments were performed on LR-0 reactor, a light water zero-power research reactor, which is operated by Research Center Řež (Czech Republic). The full-scale (in the radial direction) models (mock-ups) of VVER-440 and VVER-1000 reactors were developed and investigated on LR-0 jointly by Nuclear Research Institute Řež (Czech Republic), Škoda Nuclear Machinery Plzen (Czech Republic), and NRC Kurchatov Institute (Russia), see e.g. [2] and [3]. The top view on the VVER-1000 mock-up is presented in Fig. 1.

The core models of baffle and barrel are located inside the LR-0 aluminum tank. The VVER-1000 reactor pressure vessel (RPV) simulator and concrete radiation shielding model are located outside of LR-0 tank.

The core consists of 32 fuel assemblies of various enrichment, and the length of fuel fissile column is equal to $125 \mathrm{~cm}$. Demineralized water with diluted boric acid of a concentration of $4.7 \pm 0.1 \mathrm{~g} / \mathrm{kg}$ is used as a moderator. The moderator level is equal to $150 \mathrm{~cm}$, creating a $25 \mathrm{~cm}$ upper reflector. The criticality in this core arrangement is adjusted using 2 control clusters ( 3 absorbing $\mathrm{B}_{4} \mathrm{C}$ rods only in each cluster) in the positions marked with a " $C$ " in the Fig. 1 . Clusters are positioned at $60.4 \mathrm{~cm}$ above the bottom of the fissile column.

More details can be found in [4].

Fast neutron and gamma spectra were measured during years of investigations on the outer surface of barrel (inside the displacer), at several points inside the downcomer, on the inner and outer surfaces and at several points inside RPV model, and in the five types of neutron monitor channels in the radiation shielding model. 


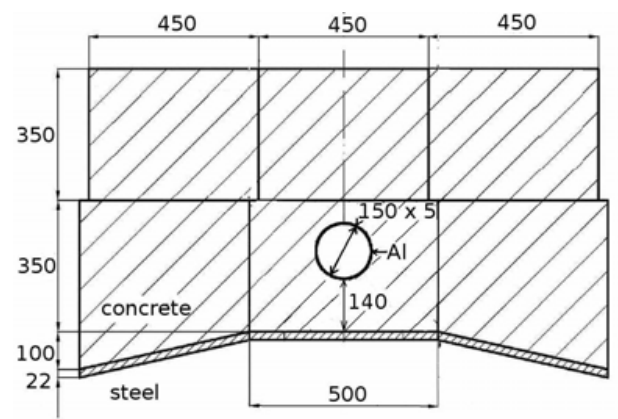

Arrangement S1

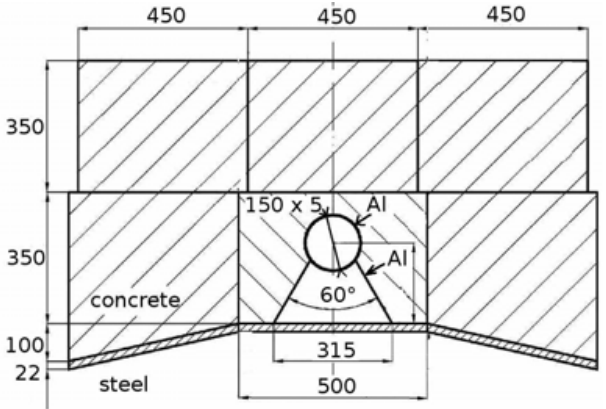

Arrangement S2

Figure 2. Selected arrangements of biological shielding simulator dimensions are in $\mathrm{mm}$.

Here we consider the last experimental results on the outer surface of barrel ("point 2"), inner ("point 3") and outer ("point 7") RPV surfaces and in two types of monitor channels. The distance between the displacer (empty steel tank) and the LR-0 tank (i.e., water gap width) is equal to $19.6 \mathrm{~cm}$.

The distance between the LR-0 tank and the shielding model was equal to $34 \mathrm{~cm}$. The RPV model (width $20 \mathrm{~cm}$ ) was moved to the shielding model for measurements at point 3 and to the LR-0 tank for measurements at point 7 , providing corresponding gaps where the neutron detector was located.

Two models of radiation shielding considered in this paper are shown in the Fig. 2. The neutron monitor channel designs in these models correspond to designs in different VVER-1000 reactors.

Information concerning composition, density and humidity of concrete in these two models of radiation shielding can be found in [1].

\section{Fast Neutron Spectra Measurements}

Neutron spectra in the energy range from 0.6 to $10 \mathrm{MeV}$ were measured by the proton-recoil method using an organic scintillator stilbene with neutron and gamma pulse shape discrimination. A $20 \times 20 \mathrm{~mm}$ stilbene crystal was used. The two-parametric spectrometric system based on analogue processing of output signals from a scintillation stilbene detector is described in [5]. Neutron spectra from 0.1 to $1.35 \mathrm{MeV}$ were measured by two hydrogen proportional counters: with hydrogen pressure $400 \mathrm{kPa}$ for measurements in energy range from $0.1 \mathrm{MeV}$ to $0.7 \mathrm{MeV}$ and with pressure $1000 \mathrm{kPa}$ for measurements from 0.25 to $1.35 \mathrm{MeV}$ [4].

The spectra obtained by both experimental methods are combined using most reliable parts of each spectrum. Both spectra were normalized to LR-0 power monitor records. The combined neutron spectrum in the energy range $0.1-10 \mathrm{MeV}$ consists of three parts: spectrum measured with hydrogen proportional counters in the range $0.1-0.8 \mathrm{MeV}$, spectrum measured with stilbene spectrometer in the range $1.2-10 \mathrm{MeV}$, and spectrum in the range $0.8-1.2 \mathrm{MeV}$, which is averaged between spectra measured by both methods.

It is necessary to mention that the procedure of spectra combination is not unambiguous and a special analysis and choice of energy intervals and algorithms for spectra combination are necessary in every specific situation. A digital neutron-gamma scintillation spectrometry, which is now developed [6-8], could potentially allow measurement of a neutron spectrum in the whole desirable neutron energy range in one measurement. In this case results would be more reliable than combined ones.

The months-long period of LR-0 power stability is crucial for spectra measurements.

Monitoring of reactor power was carried out by an independent measurement with a compensated boron chamber placed in the side region of the reactor core. The discrepancy between the proportionality 
of the monitors was estimated independently by means of measurement with a well-defined ${ }^{3} \mathrm{He}$ detector [9]. The ratio between responses of both detectors fluctuated within $1.2 \%$. Thus the conservative estimation of uncertainty of experimental values normalized by means of such monitors is not higher than $1.2 \%$.

The experimental group spectra (each group width is equal to $0.1 \mathrm{MeV}$ ) are presented in the Fig. 3 . The spectrum in each point of measurement is normalized to the LR-0 power monitor current.

The estimated experimental uncertainties depend on point of measurement, experimental conditions, number of measurements series, etc. For point 2 the uncertainties of group neutron fluxes were estimated as $1-3 \%$ in the interval from 0.1 to $4 \mathrm{MeV}$ and as $5-10 \%$ above $5 \mathrm{MeV}$. In point 3 uncertainties were estimated as $5-10 \%$ up to $6 \mathrm{MeV}$ and up to $20 \%$ at higher neutron energy. In point 7 the uncertainty level was estimated as 10-20\% in the whole energy interval. In point S1 the uncertainty was estimated as $5-10 \%$ up to $8 \mathrm{MeV}$ and $10-20 \%$ above. In point S2 the uncertainty was estimated as $2-10 \%$ up to $5 \mathrm{MeV}$ and $10-20 \%$ above.

\section{Fast Neutron Spectra Calculations}

Calculations of neutron flux at selected VVER-1000 mock-up points as well as in the biological shielding simulator were performed using the MCNPX 2.6.0 code [10] and various nuclear data libraries: ENDF/B VII.0, JEFF 3.1, JENDL 3.3, JENDL 4, ROSFOND 2009, and CENDL 3.1 [11, 12]. Nuclear data were processed with NJOY code [13].

Neutron flux in the RPV and biological shielding was calculated using a fixed source model. In this approach the beginning part of neutron evolution, comprising a detailed description of neutron origin in fuel, is described directly by means of neutron emission density. This number can be replaced by fission density due to their near proportionality. The Watt distribution of ${ }^{235} \mathrm{U}$ thermal neutron fission spectra was used for neutron source emission spectra definition because of the negligible differences in the emission spectra between the peripheral pin, the pin on the boundary with water gap and the Watt distribution. More details can be found in [14].

The pin power density distribution in the core was calculated using a defined source model, which provides a detailed description of neutron transport in the fuel, namely the origination of new neutrons [15]. The results of the calculations are used as the source description in the fixed source model mentioned in the previous paragraph.

The calculated spectra are shown in the Fig. 3 where they are compared with measured ones. The calculated group flux uncertainty increases from $0.5 \%$ at energy below $1 \mathrm{MeV}$ to $5 \%$ at approximately $5 \mathrm{MeV}$, to $10 \%$ at approximately $7 \mathrm{MeV}$, and to $25 \%$ at $10 \mathrm{MeV}$.

\section{Spectral Indices}

Spectral indices (SI) are used in reactor dosimetry for integral characterization of the neutron spectrum shape as well as in practical dosimetry. They are relative quantities reflecting the material properties of matter through which neutrons are passing. An SI is defined as a ratio of two integral neutron flux densities over different thresholds. Three SI are compared in this paper: SI(0.1/3.0) i.e., a ratio of flux density over $0.1 \mathrm{MeV}$ to flux density over $3.0 \mathrm{MeV}, \mathrm{SI}(0.5 / 3.0)$, and $\mathrm{SI}(1.0 / 3.0)$.

The experimentally determined SI are presented in the Table 1.

The presented results demonstrate a hardening of the spectrum, i.e., increasing high energy neutrons, in water and concrete and softening in steel.

The comparison of the calculation and measured SI is in the Table 2 where values (calculated SI/measured SI - 1)\% are shown. The estimated uncertainties of calculated SI are not higher than $2 \%$. 


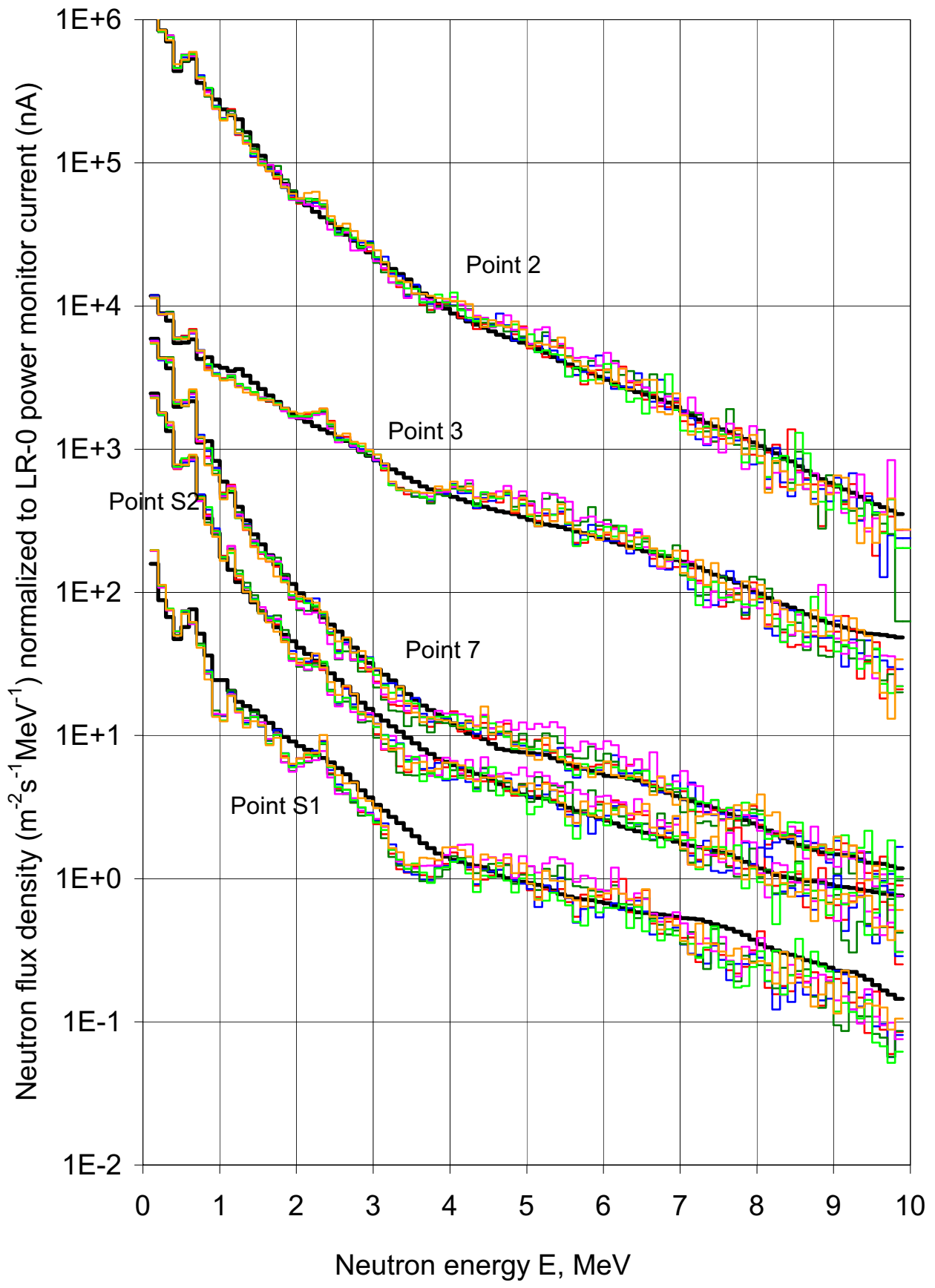

$\begin{array}{llll}\text { - Experiment } & \text { - ENDF / B VII.0 - JEFF } 3.1 & \text { - JENDL } 3.3 \\ \text { - JENDL } 4 & \text { - ROSFOND 2009 - CENDL } 3.1\end{array}$

Figure 3. Measured and calculated neutron spectra at different points in the mock-up. 
Table 1. Measured SI.

\begin{tabular}{|c|r|r|r|r|r|}
\hline \multirow{2}{*}{$\begin{array}{c}\text { Threshold } \\
\text { energies, MeV }\end{array}$} & \multicolumn{5}{|c|}{ Points in mock-up } \\
\cline { 2 - 6 } & 2 & 3 & 7 & S1 & S2 \\
\cline { 2 - 6 } & & \multicolumn{5}{|c|}{ Spectral indices } \\
\hline $0.1 / 3$ & $23.0 \pm 1.9 \%$ & $6.57 \pm 4.4 \%$ & $58.60 \pm 4.2 \%$ & $14.60 \pm 5.0 \%$ & $44.72 \pm 2.3 \%$ \\
\hline $0.5 / 3$ & $13.08 \pm 1.8 \%$ & $4.64 \pm 4.3 \%$ & $24.4 \pm 4.4 \%$ & $8.24 \pm 5.0 \%$ & $17.58 \pm 2.4 \%$ \\
\hline $1 / 3$ & $6.61 \pm 1.4 \%$ & $3.29 \pm 4.2 \%$ & $8.46 \pm 4.7 \%$ & $4.56 \pm 4.2 \%$ & $6.22 \pm 2.1 \%$ \\
\hline
\end{tabular}

Table 2. Comparison of calculated and measured SI, (C/M-1)\%.

\begin{tabular}{|c|c|c|c|c|c|c|c|}
\hline \multirow{3}{*}{ Point } & \multirow{3}{*}{ SI } & \multicolumn{6}{|c|}{ Library } \\
\hline & & $\begin{array}{c}\text { ENDF/B } \\
\text { VII.0 }\end{array}$ & JEFF 3.1 & JENDL 3.3 & JENDL 4 & ROSFOND 2009 & CENDL 3.1 \\
\hline & & & & & $\overline{\mathrm{C} / \mathrm{M}-1) \%}$ & & \\
\hline \multirow{4}{*}{2} & $0.1 / 3$ & & -1.3 & -0.1 & -4.1 & -1.1 & -5.6 \\
\hline & & 2.6 & & & & & \\
\hline & $0.5 / 3$ & 1.6 & -2.0 & -1.1 & -5.6 & -1.3 & -6.6 \\
\hline & $1 / 3$ & -4.0 & -5.9 & -1.7 & -9.1 & -6.4 & -8.8 \\
\hline \multirow[t]{3}{*}{3} & $0.1 / 3$ & 1.6 & 2.4 & -5.0 & -7.5 & 0.2 & -3.7 \\
\hline & $0.5 / 3$ & 0.2 & 0.3 & -6.5 & -8.6 & -0.8 & -4.6 \\
\hline & $1 / 3$ & -2.4 & -2.0 & -7.6 & -9.4 & -3.1 & -5.7 \\
\hline \multirow[t]{3}{*}{7} & $0.1 / 3$ & 2.6 & -2.1 & 5.1 & -11.1 & -2.4 & 0.6 \\
\hline & $0.5 / 3$ & 0.1 & -4.0 & -0.6 & -14.0 & -2.3 & -4.0 \\
\hline & $1 / 3$ & -6.4 & -7.5 & -3.6 & -17.0 & -10.2 & -9.4 \\
\hline \multirow[t]{3}{*}{ S1 } & $0.1 / 3$ & 29.6 & 34.1 & 25.1 & 8.0 & 36.9 & 14.4 \\
\hline & $0.5 / 3$ & 15.2 & 19.6 & 11.6 & -2.9 & 20.6 & 1.7 \\
\hline & $1 / 3$ & 4.2 & 8.0 & 1.4 & -11.5 & 7.2 & -5.3 \\
\hline \multirow[t]{3}{*}{ S2 } & $0.1 / 3$ & 12.2 & 10.1 & 14.4 & -1.7 & 14.6 & 8.6 \\
\hline & $0.5 / 3$ & 14.5 & 12.6 & 13.2 & -0.6 & 19.6 & 7.8 \\
\hline & $1 / 3$ & 9.4 & 9.2 & 13.3 & -2.0 & 10.7 & 4.8 \\
\hline
\end{tabular}

The discrepancies over $\pm 10 \%$ are highlighted by grey. They are in concrete with all libraries and behind the RPV with JENDL 4. In other cases calculations with all libraries give reasonable agreement with experiment.

\section{Neutron Flux Density Attenuation Factors}

Attenuation factors (AF) are determined as ratios of integral flux densities at different points in the mock - up. In this paper AF are determined for integral flux densities over thresholds of $0.1,0.5,1$, and $3 \mathrm{MeV}$. Flux attenuations are determined for the downcomer (AF 2/3), RPV simulator (AF 3/7), space between RPV outer surface and monitor channel (AF 7/S1 and 7/S2). The integral flux ratios S2/S1 characterize an effect of monitor channel placement and geometry.

Experimentally determined neutron flux density AF with related uncertainties are presented in the Table 3.

In water and concrete an $\mathrm{AF}$ decreases with increasing threshold neutron energy. In steel the dependence is opposite. 
Table 3. Measured neutron flux density AF.

\begin{tabular}{|c|c|c|c|c|c|}
\hline \multirow{2}{*}{$\begin{array}{c}\text { Threshold } \\
\text { energy, MeV }\end{array}$} & \multicolumn{5}{|c|}{ Attenuation factors } \\
\cline { 2 - 6 } & $2 / 3$ & $3 / 7$ & $7 / \mathrm{S} 1$ & $7 / \mathrm{S} 2$ & $\mathrm{~S} 2 / \mathrm{S} 1$ \\
\hline 0.1 & $61.9 \pm 3.4 \%$ & $4.26 \pm 3.6 \%$ & $32.9 \pm 4.8 \%$ & $2.62 \pm 2.4 \%$ & $12.6 \pm 4.6 \%$ \\
\hline 0.5 & $49.8 \pm 3.1 \%$ & $7.26 \pm 3.7 \%$ & $24.2 \pm 5.0 \%$ & $2.76 \pm 2.9 \%$ & $8.78 \pm 4.6 \%$ \\
\hline 1 & $35.5 \pm 2.8 \%$ & $14.8 \pm 3.9 \%$ & $15.2 \pm 4.4 \%$ & $2.71 \pm 3.0 \%$ & $5.60 \pm 3.6 \%$ \\
\hline 3 & $17.6 \pm 3.4 \%$ & $38.1 \pm 4.9 \%$ & $8.18 \pm 4.4 \%$ & $1.99 \pm 4.1 \%$ & $4.11 \pm 3.0 \%$ \\
\hline
\end{tabular}

Table 4. Comparison of calculated and measured AF, (C/M-1)\%.

\begin{tabular}{|c|c|c|c|c|c|c|c|}
\hline \multirow[b]{2}{*}{$\mathrm{AF}$} & \multirow[b]{2}{*}{$\begin{array}{c}\text { Threshold } \\
\text { energy }\end{array}$} & \multicolumn{6}{|c|}{ Library } \\
\hline & & $\begin{array}{c}\text { ENDF/B } \\
\text { VII.0 }\end{array}$ & JEFF 3.1 & JENDL 3.3 & JENDL 4 & $\begin{array}{c}\text { ROSFOND } \\
2009\end{array}$ & $\begin{array}{c}\text { CENDL } \\
3.1\end{array}$ \\
\hline & & $\mathrm{MeV}$ & & & $(\mathrm{C} / \mathrm{M}-1) \%$ & & \\
\hline \multirow{4}{*}{$2 / 3$} & 0.1 & 19.7 & 19.5 & 13.5 & 12.8 & 19.3 & 16.6 \\
\hline & 0.5 & 20.1 & 21.2 & 14.2 & 12.5 & 20.4 & 16.6 \\
\hline & 1.0 & 16.6 & 19.2 & 14.9 & 9.3 & 16.9 & 15.1 \\
\hline & 3.0 & 19.0 & 24.6 & 8.4 & 9.3 & 21.5 & 19.6 \\
\hline \multirow{4}{*}{$3 / 7$} & 0.1 & -8.0 & -9.0 & -6.5 & -8.2 & -9.2 & -6.4 \\
\hline & 0.5 & -7.3 & -9.5 & -3.2 & -6.6 & -10.6 & -3.4 \\
\hline & 1.0 & -3.3 & -8.0 & -1.2 & -3.8 & -4.8 & 1.6 \\
\hline & 3.0 & -8.0 & -9.0 & -6.5 & -8.2 & -9.2 & -6.4 \\
\hline \multirow{4}{*}{$7 / \mathrm{S} 1$} & 0.1 & -33.3 & -32.6 & -31.5 & -32.7 & -33.8 & -24.9 \\
\hline & 0.5 & -26.7 & -25.6 & -27.2 & -27.4 & -24.5 & -19.1 \\
\hline & 1.0 & -24.3 & -20.9 & -22.4 & -23.3 & -22.1 & -18.3 \\
\hline & 3.0 & -15.6 & -7.5 & -18.3 & -18.1 & -6.9 & -14.4 \\
\hline \multirow{4}{*}{$7 / \mathrm{S} 2$} & 0.1 & 0.1 & 1.5 & 1.8 & 1.6 & 0.4 & 4.1 \\
\hline & 0.5 & -3.9 & -2.1 & -2.3 & -2.3 & -3.3 & 0.6 \\
\hline & 1.0 & -6.1 & -3.0 & -5.4 & -4.6 & -4.0 & -2.5 \\
\hline & 3.0 & 10.0 & 14.7 & 11.3 & 12.8 & 18.4 & 12.9 \\
\hline \multirow{4}{*}{$\mathrm{S} 2 / \mathrm{S} 1$} & 0.1 & -33.7 & -33.8 & -33.0 & -34.0 & -34.3 & -28.1 \\
\hline & 0.5 & -23.8 & -24.1 & -25.6 & -25.7 & -22.1 & -19.7 \\
\hline & 1.0 & -19.2 & -18.3 & -17.9 & -19.5 & -18.7 & -16.0 \\
\hline & 3.0 & -23.2 & -19.3 & -26.6 & -27.4 & -21.4 & -24.2 \\
\hline
\end{tabular}

A comparison of calculated neutron flux density attenuation factors with measured ones is presented in the Table 4, where (C/M-1)\% values are shown.

The estimated uncertainties of calculated AF are not higher than $2 \%$.

The discrepancies over $10 \%$ are highlighted by grey. As seen here calculation with every library overestimates attenuations factors in water and underestimates in the RPV and concrete compared with the experiment.

\section{Discussion}

A new experiment was carried out in the VVER-1000 mock-up on the LR-0 reactor. In this experiment neutron spectra at representative points were measured in the energy range from 0.1 to $10 \mathrm{MeV}$ 
combining the proton-recoil method with organic scintillator stilbene in the neutron energy region 0.6$10 \mathrm{MeV}$ and two hydrogen proportional counters in the region $0.1-1.35 \mathrm{MeV}$.

Neutron spectra, SI, and AF were calculated using code MCNPX 2.6.0 and nuclear data libraries ENDF/B VII.0, JEFF 3.1, JENDL 3.3, JENDL 4, ROSFOND 2009, and CENDL 3.1.

On the barrel surface all calculated spectra agree with measured ones within the summarized uncertainty of measurement and calculation (statistical).

On the inner surface of the RPV discrepancies between shapes of calculated and measured spectra have the same character for all libraries: calculated group fluxes are less than measured ones between approx. 1 and 2, 3 and $4 \mathrm{MeV}$ and more between 2 and 3, 4 and $6 \mathrm{MeV}$. The discrepancies are minimal for library CENDL 3.1 and maximal for library JENDL 4 (in the interval 4-7 MeV).

On the outer surface of the RPV there are noticeable discrepancies between shapes of calculated and measured spectra between 3 and $4 \mathrm{MeV}$ for JENDL 3.3 and between 4 and 7 for JENDL 4 . For other libraries the discrepancy between calculated and measured spectra shapes is comparable with related uncertainties.

Results are approximately the same in channel S2 in the concrete shielding model.

In channel $\mathrm{S} 1$, a concrete calculation underestimates neutron flux around $2 \mathrm{MeV}$, between 3 and $4 \mathrm{MeV}$, and over $7 \mathrm{MeV}$ and overestimates between 5 and $6 \mathrm{MeV}$.

Calculated SI agree with experiment data reasonably well for all libraries with the exception of channels in concrete and JENDL 4 results on the outer RPV surface (Table 2). JENDL 3.3 gives better agreement but JENDL 4 and CENDL 3.1 give worse agreement on the barrel surface. On the inner RPV surface the agreement is worse in the case of JENDL 3.3 and JENDL 4. The calculation/experiment discrepancy on the outer RPV surface is significantly higher in the case of JENDL 4 than in JENDL 3.3 and other libraries. Similar results concerning calculation of neutrons transport through iron using JENDL 3.3 and JENDL 4 were reported in [16].

Calculations with all libraries approximately to the same extent overestimate AF in water and underestimate in RPV and concrete (Table 4). Better agreement of calculated and measured AF in water is found in JENDL 3.3 and JENDL 4. Better agreement of calculated and measured AF in RPV is found in JENDL 3.3 and CENDL 3.1.

The experiments and calculations were conducted under contract No. Rea/11/34/996-10/INR between Research Centre Řež ltd. and National Research Centre "Kurchatov Institute" (Moscow, Russia), have been funded also by the Research Centre Řež ltd. under Czech Republic Ministry of Education, Youth and Sport project MSM 2672244501, under the SUSEN Project CZ.1.05/2.1.00/03.0108 realized in the framework of the European Regional Development Fund (ERDF) and University of Defence CR Specific research support.

\section{References}

[1] S. Zaritskiy, A. Egorov, B. Ošmera, M. Mařik, V. Rypar, F. Cvachovec, A. Kolros, "Neutron and Gamma Fields Investigations in the VVER1000 Mock-up Concrete Shielding on the Reactor LR-0”, Journal of ASTM International, vol. 9, No. 3, 2012, Paper ID JAI103995

[2] B. Ošmera, S. Zaritsky, "Review of Experimental Data for WWER Reactor Pressure Vessel Dosimetry Benchmarking”, in Reactor Dosimetry in the 21st Century, Proc. 11th Int. Symp. on Reactor Dosimetry, edited by Jan Wagemans, Hamid Aït Abderrahim, Pierre D'hondt, Charles De Raedt. World Scientific, Singapore, 2003, pp. 689-696

[3] S. Zaritsky, B. Ošmera, F. Cvachovec, M. Mařik, S. Pošta, V. Rypar, D. Riazanov, V. Lichadeev, A. Kolros, and Z. Matěj, "Neutron and Gamma Spectrometry in the Research Reactor LR-0", in IEEE Proceedings of ANIMMA, IEEE, Washington D.C., 2009, ISBN number: 978-1-42445208-8, IEEE Catalogue Number: CFP09241-CDR 
[4] B. Ošmera, S. Zaritski, "WWER-1000 Benchmarks in LR-0 Experimental Reactor”, UJV Řež Report No.12993-R, pp 1-106, 2008

[5] Z. Bureš, J. Cvachovec, F. Cvachovec, P. Čeleda, B. Ošmera, "Multiparameter Multichannel Analyser System for Characterization of Mixed Neutron-Gamma Field in the Experimental Reactor LR-0", in Reactor Dosimetry in the 21st Century, Proc. 11th Int. Symp. on Reactor Dosimetry, edited by Jan Wagemans, Hamid Aït Abderrahim, Pierre D’hondt, Charles De Raedt. World Scientific, Singapore, 2003, pp. 194-201

[6] M.V. Prokuronov, A.N. Shabalin, "A Digital Method for Pulse-Shape Discrimination between Neutrons and Gamma-Rays at a High Counting Rate and a Low Energy of Detected Radiation", Instruments and Experimental Techniques (Springer), vol. 50, issue 3, May 2007, pp. 311-324

[7] M. Prokuronov, P. Rudnev, V. Sevastyanov, "Spectrometer of Neutron and Gamma Irradiation Based on Digital Methods of Signal Registration and Processing", Modern electronics, issue 11, 2011, pp. 38-41 (in Russian), www.soel.ru

[8] F. Cvachovec, M. Čech, M. Košt'ál, Z. Matěj, F. Mravec, V. Přenosil, M. Veškrna, "Digital Spectrometric System for Characterization of Mixed Neutron-Gamma Field in the Experimental Reactor LR-0", ISRD-15, paper 128

[9] A. Kolros, O. Huml, M. Kř́íz, J. Kos, "Equipment for Neutron Measurements at VR-1 Sparrow Training Reactor", Applied Radiation and Isotopes, vol. 68, issue 4-5, April-May 2010, pp 570574

[10] D.B. Pellowitz, “MCNPX User’s Manual, version 2.6.0”, Los Alamos Report No. LA CP 02 408, 2007

[11] JEFF 3.1, JENDL 3.3, JENDL 4, CENDL 3.1., ROSFOND 2009 obtained from www.nndc. bnl.gov, accessed 24.6.2010

[12] ENDF/B VII.0, RSICC, obtained in package D00226MNYCP01, 2008

[13] R.E. MacFarlane, D.W. Muir, "The NJOY Nuclear Data Processing System, Version 91", LA12740-M, 1994

[14] Michal Košt'ál, František Cvachovec, Vojtěch Rypar, Vlastimil Juříček, "Calculation and Measurement of Neutron Flux in the VVER-1000 Mock-up on the LR-0 Research Reactor", Annals of Nuclear Energy, 40 (2012) pp 25-34

[15] M. Košt’ál, V. Rypar, M. Švadlenková, "The Pin Power Distribution in the VVER-1000 Mock-up on the LR-0 Research Reactor", Nucl. Eng. and Design, 242 (2012) 201-214

[16] Michal Košt'ál, František Cvachovec, Jiři Cvachovec, Bohumil Ošmera, Wolfgang Hansen, "Neutron and Photon Transport in Fe with the Employment of TENDL 2009, CENDL 3.1, JENDL 4 and JENDL 4 Evolution from JENDL 3.3 in Case of Fe", Nucl. Eng. and Design, 249 (2012) 275-286 